\title{
Würgefeigen: Baumwürger und ihr geheimes Sexualleben. „Mordlüsterne“ Pflanzen oder Lebenskünstler?
}

Herwig Zahorka

\begin{abstract}
Many species of the genus Ficus (Moraceae, mulberry family) that grow in the tropics and subtropics, form aerial roots. The fruits of these species are an important food source for birds, monkeys and other animals. Through the feces of these animals, a seed can come into the substrate of a branch fork and germinate here. The epiphytic plantlet forms aerial roots. When they reach the ground, they develop into a network of stems, which can eventually encase the support tree all around. It is overgrown, strangled and dies. When its wood is destroyed by termites, a chimney-like cavity remains inside the choking tree. The secret sexual life of the Ficus species takes place inside the unspectacular small closed inflorescence. It is a small fleshy hollow body with a large number of male and two different female flowers on its inner surface. Pollination inside is performed by tiny wasps that penetrate through a small opening. Almost every Ficus species has a symbiotic relationship to a specific wasp species.
\end{abstract}

\section{Zusammenfassung}

Viele Arten der Gattung Ficus (Moraceae, Maulbeergewächse) in den Tropen und Subtropen bilden Luftwurzeln. Feigenfrüchte sind eine wichtige Nahrungsgrundlage für Vögel, Affen und andere Tiere. Durch den Kot dieser Tiere kann ein Same in das Substrat einer Astgabel kommen und hier keimen. Das epiphytische Pfänzchen bildet Luftwurzeln. Wenn diese den Boden erreichen, entwickeln sie sich zu einem Geflecht von Stämmchen, die schließlich den Trägerbaum rundherum dicht ummanteln können. Er wird überwachsen, „erwürgt“ und stirbt ab. Wenn sein Holz von Termiten zerstört ist, bleibt im Inneren ein kaminartiger Hohlraum übrig. Das geheime Sexualleben der Ficus-Arten spielt sich im Inneren der unspektakulär kleinen geschlossenen Fruchtstände ab. Es handelt sich um kleine fleischige Hohlkörper, an dessen innerer Oberfläche sich eine große Zahl von männlichen und zwei verschiedenen Typen weiblicher Blüten befindet. Die Bestäubung im Inneren findet durch winzige Feigenwespen statt, die durch eine kleine Öffnung in den Blütenstand eindringen können. Fast jede Ficus-Art ist auf die Bestäubung durch eine ganz spezielle Wespenart angewiesen.

\section{Die Baumwürger der Gattung Ficus}

Weil fast alle Baumwürger der Gattung Ficus angehören, werden Feigenbäume oft ganz allgemein als Würgefeigen bezeichnet. Die Gattung Ficus umfasst je nach Auffassung bis 1000 Arten, wobei es sich um Bäume oder Sträucher handelt. Sie sind in tropischen und subtropischen Regionen Asiens, Amerikas und Afrikas zu Hause. Besonders häufig finden sich Ficus-Arten in Regenwäldern. Alle enthalten einen weißen Milchsaft. Nicht wenige sind in der Lage, Luftwurzeln zu bilden.

Ihre generative Vermehrung ist von winzigen Feigenwespen (Agaonidae) abhängig. Viele Arten benötigen für eine erfolgreiche Bestäubung ihre ganz spezielle Feigenwespe. Diese gegenseitige Abhängigkeit wird Mutualismus genannt. Das, was wohl die meisten als Früchte der Feigen bezeichnen, sind eigentlich Fruchtverbände. Diese sind meist erbsen- bis kirschgroß, rund oder birnenförmig. Sie sind eine wichtige Nahrungsgrundlage für Vögel, Affen und Fledertiere. Von vielen Ficus-Arten

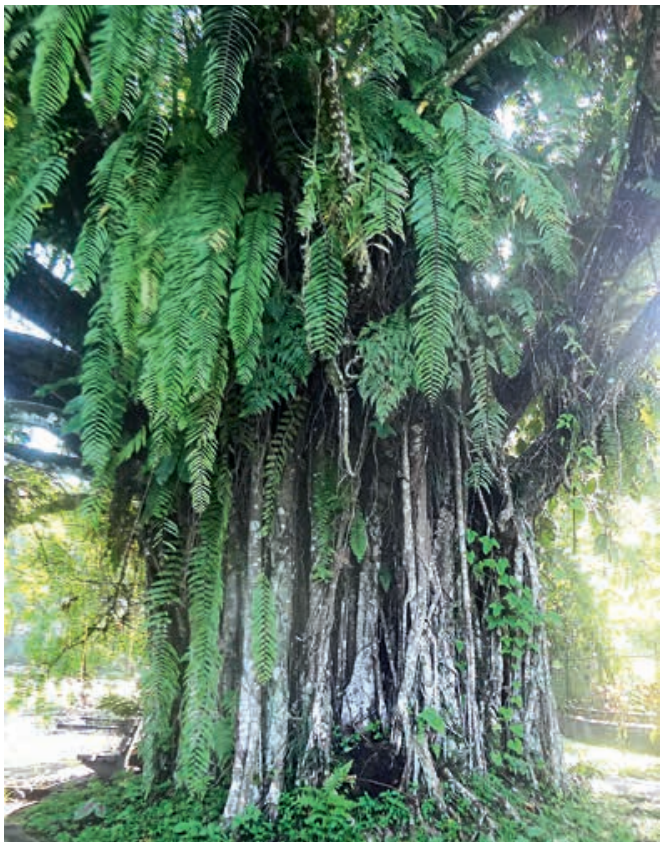

Abb. 1: Ein stattliches Exemplar von Ficus religiosa in Indonesien, etwa 60 Jahre alt, mit zahlreichen Luftwurzeln. (Foto: H. ZAHORKA) 


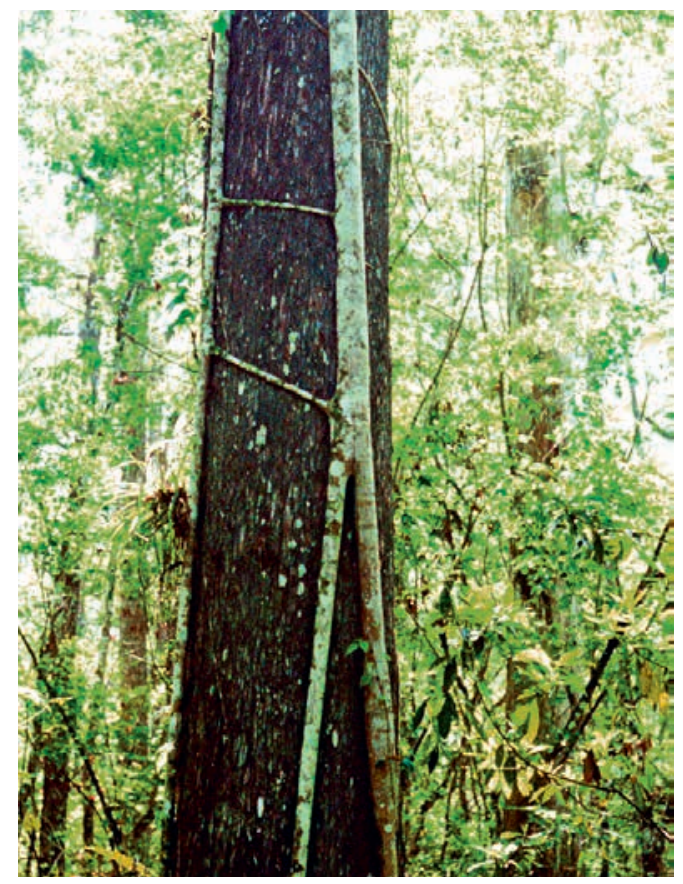

Abb. 2: Die in Florida heimische Würgefeige Ficus aurea hat erste Wurzeln um den Wirtsbaum geschlungen.

(Foto: H. Steinecke)

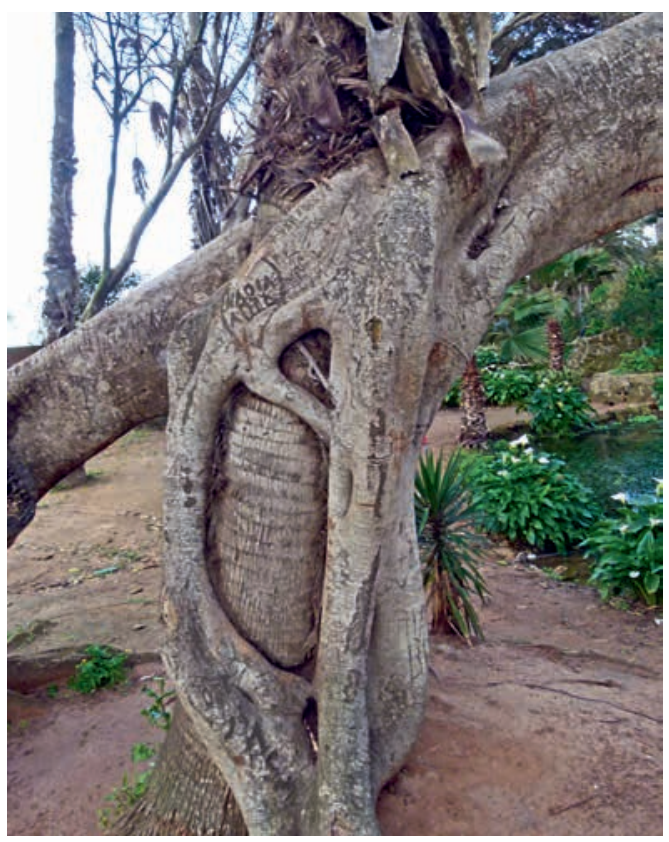

Abb. 3: Eine Dattelpalme in einem marokkanischen Park wird von Wurzeln einer Würgefeige umgeben. Da der Palmenstamm nicht in die Breite wächst, wird die Palme so bald nicht erwürgt werden. (Foto: H. STEInecke) werden aber auch Blätter und Rinden in der Ethnomedizin verwendet.

\section{Die Entwicklung zur Würgefeige}

Vögel oder Affen, die Feigenfrüchte gefressen haben, scheiden irgendwann wieder die in ihnen enthaltenen Kerne mit den Samen aus. Diese passieren den Darm der Tiere, ohne dabei ihre Keimfähigkeit zu verlieren. Wenn die Samen in einer Astgabel eines beliebigen Trägerbaumes landen, keimen dort die Samen und kann der Keimling „Fuß fassen“. Es entwickelt sich zunächst ein kleines epiphytisches Pflänzchen mit einigen Blättern und einer feinen Luftwurzel. Wenn diese den Boden erreicht, verzweigt sie sich rasch und ernährt die zum Semiepiphyten herangewachsene Feige. Diese entwickelt nun immer mehr derartige Luftwurzeln, was zu einem schnellen Wachstum der jungen Würgefeige führt. Die fadenförmigen Luftwurzeln fühlen sich hart und „hölzern“ an. Bevor sie den Boden erreichen, ist ihre Farbe oft rötlich, seltener grün. Durch diesen „Angriff aus der Luft" kann das Schicksal des Wirtsbaumes besiegelt sein.

Die Luftwurzeln verdicken sich schnell, wachsen an Berührungspunkten zusammen, werden stammförmig und bilden eine netzartige Struktur rund um den Wirtsbaum. Mehrere Schichten von zu Stämmchen gewordenen Luftwurzeln entstehen übereinander und verfilzen sich. Schließlich können sie den gesamten Wirtsbaum dicht anliegend wie ein Panzer umwachsen und sein Dickenwachstum verhindern. Der Wirtsbaum ist „eingesargt“. Der Würger wird später zu einem vielstämmigen Baum, der den Wirt ringförmig wie ein Korsett umgibt.

Dann überwächst auch die Laubkrone des Würgers diejenige des Wirtsbaums und beschattet dessen Blätter in existenzbedrohender Weise. Die dichten Wurzeln umrunden den Wirt und hungern ihn aus. Sie breiten sich auch oberflächlich bis zu zehn Meter weit vom Hauptstamm aus.

Der Wirtsbaum stirbt schließlich infolge von Licht- und Nahrungsmangel ab. Er wird regelrecht erwürgt. Das Totholz wird in den Tropen und Subtropen von Termiten, anderen Insekten und Pilzen schnell abgebaut. Dadurch entsteht innerhalb des 
Würgers ein kaminartiger Hohlraum. Der Würger kann sich weiter zu einem verflochtenen, weiträumigen und wirren „Baumgebirge“ entwickeln. Bei der Rodung des Regenwaldes für Ölpalmplantagen bleiben diese Baumriesen stehen, weil sie kaum gefällt werden können.

Alle Baumarten, die Luftwurzeln bilden, können zu Würgebäumen werden, wenn ihre Samen auf einem Wirtsbaum keimen und die Luftwurzeln dieser Epiphyten den Boden erreichen. Die meisten Ficus-Arten in den Tropen sind potenzielle Würgefeigen. Dass der Wirtsbaum dabei eingeht, scheint aber nicht immer der Fall.

Nachfolgend werden einige Feigenarten genannt, die sich zu Würgern entwickeln können.

Ficus altissima (Hohe Feige), F. aurea (Florida-Würgefeige), Ficus barbata (Bartfeige), F. benghalensis, F. burtt-davyi, F. citrifolia (Bartfeige), F. craterostoma, F. macrophylla, F. microcarpa (Lorbeer-Feige), F. obliqua, F. tinctoria, F. virens, F.watkinsiana.
Einige Würgefeigen aus Südostasien werden auch als Zierpflanzen gehalten oder in wärmeren Regionen als Park- und Straßenbäume gepflanzt, darunter z. B. Ficus religiosa, F. elastica, F. benjamina und F. microcarpa. Man muss gar nicht so weit reisen, um sogar in Europa riesige Würgefeigen mit prächtigen Luftwurzeln zu sehen. Bekannte große Exemplare von F. macrophylla stehen im Botanischen Garten in Orotava (Teneriffa) oder im Botanischen Garten in Palermo (Sizilien). Auch in Marokko oder auf Madeira werden in Parks und Gärten immer mal wieder Feigenbäume gepflanzt. Natürlich werden in den Gewächshäusern des Palmengartens auch einige Ficus-Arten kultiviert. Ein großes Exemplar mit vielen langen Luftwurzeln wächst auf der Empore des Palmenhauses in der Nähe des Kakadu-Käfigs. Besonders während der Winterlichter, wenn der Garten und auch das Palmenhaus illuminiert werden, wirkt dieser Vorhang aus Luftwurzeln ausgesprochen imposant.

Eigene Luftwurzeln werden dem Mutterbaum natürlich nicht gefährlich, sondern tragen nur zur Vielstämmigkeit des Mutterbaumes bei. Selbst auf

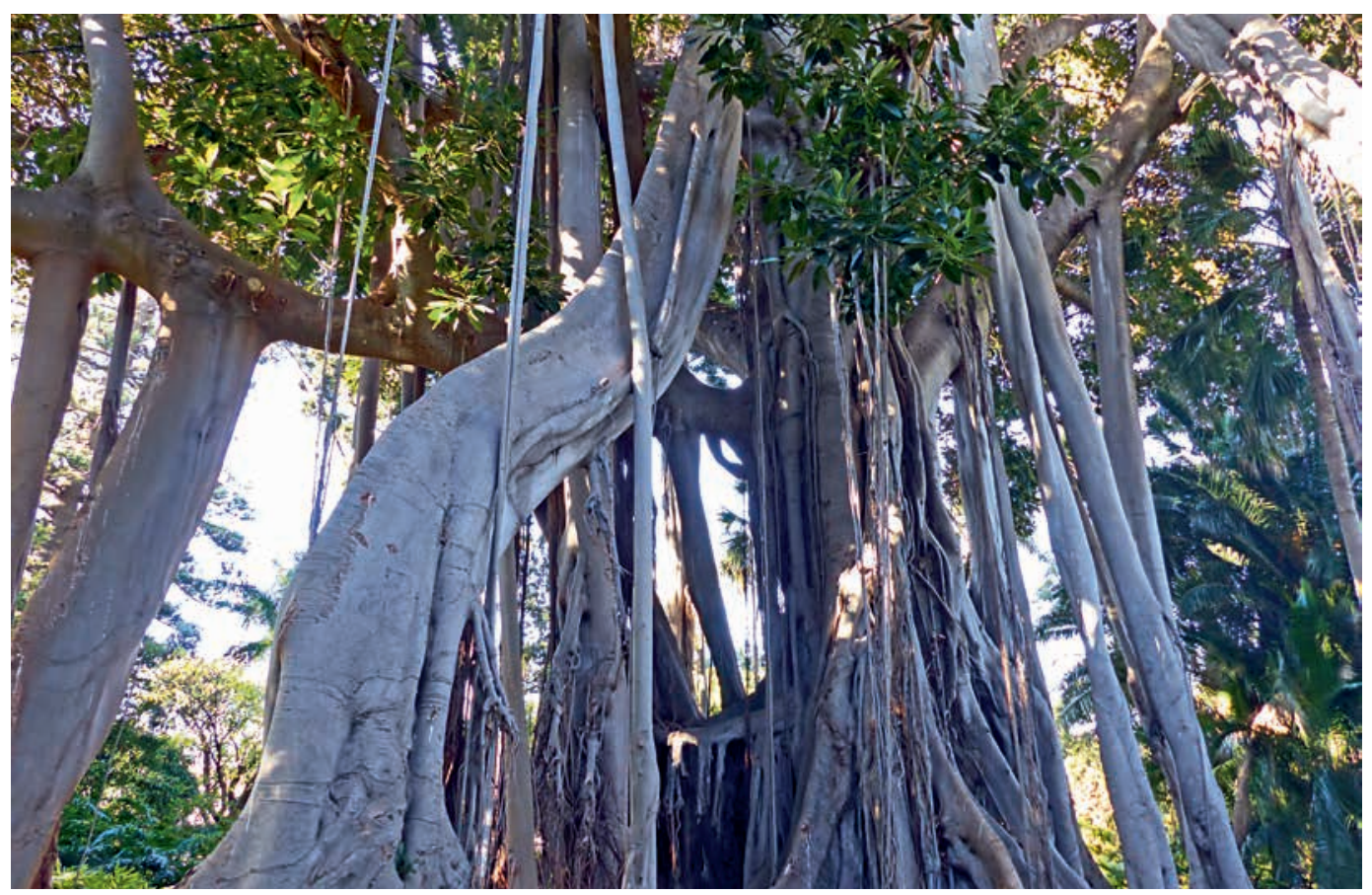

Abb. 4: Ausladendes Wurzelsystem einer alten Würgefeige, hier Ficus macrophylla f. columnaris im Botanischen Garten von Orotava auf Teneriffa. (Foto: H. STEINEcke) 


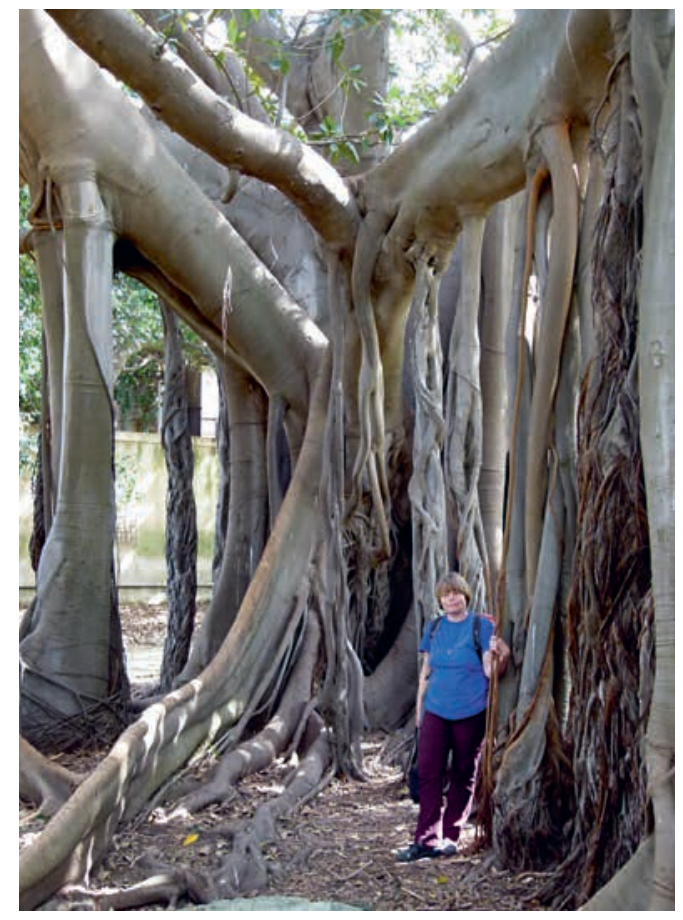

Abb. 5: Ähnlich wie in einer Tropfsteinhöhle fühlt man sich zwischen den Wurzeln einer im Jahr 1845 im Botanischen Garten von Palermo gepflanzten Würgefeige (Ficus macrophylla). (Foto: P. SCHUBERT)

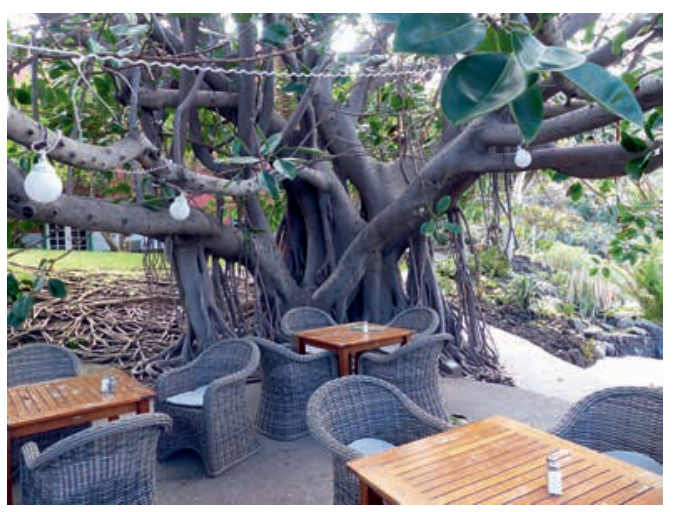

Abb. 6: Etwas größer als im Wohnzimmer: Ein schöner Sitzplatz unter einem Gummibaum (Ficus elastica) in einer Hotelanlage auf La Palma. (Foto: H. Steinecke)

Würgebäumen können wieder neue junge Würgefeigen wachsen.

Auch Vertreter anderer Gattungen können sich zu Baumwürgern entwickeln wie beispiels-

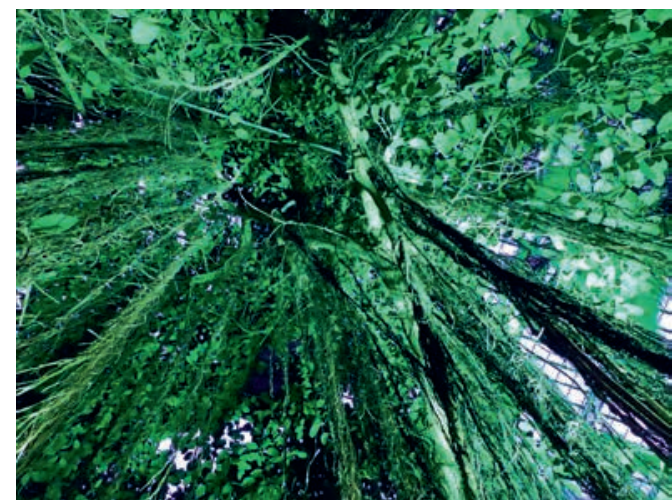

Abb. 7: Fast wie ein Blick in den tropischen Regenwald wirkt diese Perspektive in den Luftwurzelbehang einer Feige im Palmenhaus, grün illuminiert während der Winterlichter. (Foto: H. STEInecke)

weise Clusia-Arten. In seltenen Fällen kann auch der berüchtigte Giftbaum Antiaris toxicaria (wie auch Ficus ein Maulbeergewächs) Luftwurzeln ausbilden. Ein altes Exemplar des Giftbaumes steht im Botanischen Garten Bogor in Indonesien. Einige Luftwurzeln dieses Baumes haben sich schon zu dicht anliegenden, kräftigen Nebenstämmen entwickelt. Kletterpflanzen nutzen hier ihre Chance. Aus dem getrockneten Latex dieses Baumes bereiten übrigens die indigenen Dayak-Stämme Borneos ihr tödliches Gift für ihre Blasrohrpfeile (ZAHORKA 2000, 2006).

\section{Würgefeigen als gepflanzte Solitäre}

Die Vermehrung von Bäumen der Gattung Ficus erfolgt wegen der Winzigkeit ihrer Steinfrüchte (das sind die Kerne aus den „Früchten“) fast immer vegetativ mittels Ableger und Stecklingen. Beliebt in Süd- und Südostasien ist die Vermehrung von Ficus religiosa, weil unter diesem Baum angeblich Siddhartha Gautama meditierte und dabei 515 v. Chr. seine Erleuchtung zum Buddha erfuhr. Einen derartigen Bodhi tree $($ Bodhi $=$ Erwachen $)$ pflanzte der erste Präsident Indonesiens, SukarNO, vor etwa 60 Jahren im Botanischen Garten Bogor. Die heutige vielstämmige Wuchsform ist typisch für eine Würgefeige.

Bäume der Gattung Ficus können sehr alt werden. Auf Java gibt es einen tausendjährigen Ficus religiosa-Baum, der einst vor einem früheren Hindu-Heiligtum gepflanzt wurde. Heute befindet 


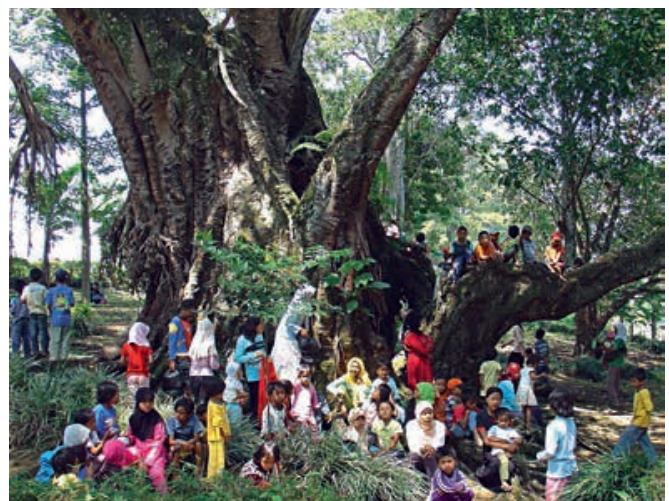

Abb. 8: Ein rund tausendjähriges Exemplar von Ficus religiosa an einem ehemaligen Hindu-Heiligtum. (Foto: H. ZaHorka)

sich hier der kleine deutsche Soldatenfriedhof Arca Domas aus dem Zweiten Weltkrieg, auf dem 10 deutsche U-Boot-Fahrer der Monsun-Flotte ihre letzte Ruhe gefunden haben.

Früher wurden Exemplare von F. religiosa und F. benghalensis häufig als Schattenbäume entlang von Straßen gepflanzt. Sie haben sich im Laufe von vielen Jahrzehnten mit Hilfe ihrer Luftwurzeln oft zu gigantischen Baumgestalten entwickelt und überschirmen den Verkehr mit einer breiten Gardine von Luftwurzeln, die den Boden nie erreicht. Davon ist man längst abgekommen, weil die flach streichenden Wurzeln im Laufe der Zeit den angrenzenden Bürgersteig aufbrechen.

\section{Das geheime Sexualleben der Feigenbäume}

Feigen bilden rundliche bis birnenförmige, geschlossene Blütenstände. Aus diesen entwickelt sich ein ganz spezieller Fruchtverband, der hier Sykonium genannt und im Volksmund als die Feigenfrucht bezeichnet wird. Es handelt sich um einen fleischigen Hohlkörper, der auf seiner inneren Oberfläche hunderte von winzigen Blüten trägt. Es gibt männliche und zwei verschiedene Typen weiblicher Blüten, nämlich solche mit langem Griffel und solche mit kurzem Griffel. Die weiblichen Blüten mit kurzem Griffel sind steril und werden auch Gallenblüten genannt.

Fast jede Ficus-Art hat einen speziellen Bestäuber, wobei es sich um winzige, rund $2 \mathrm{~mm}$

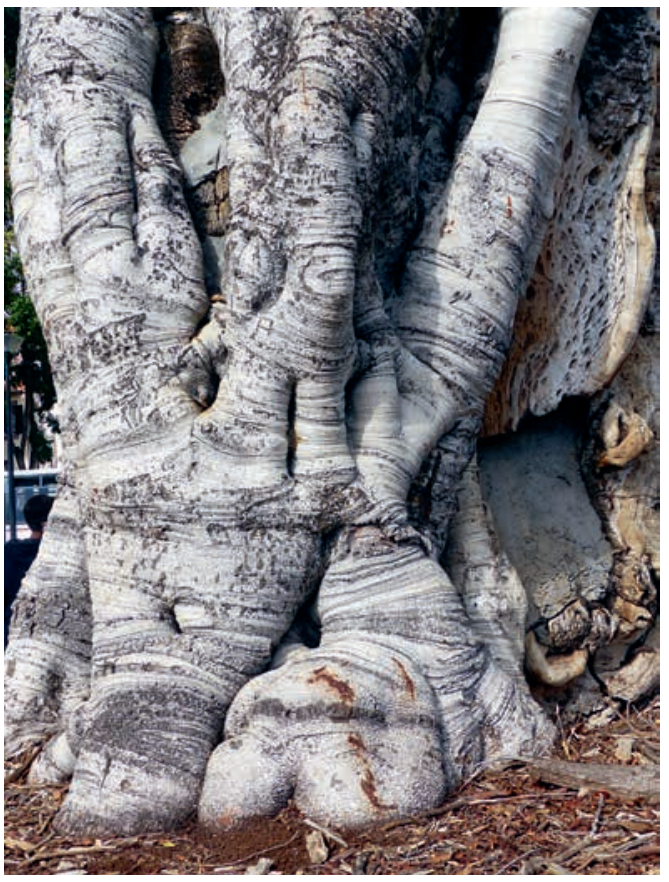

Abb. 9: Die Stämme von älteren Straßenbaum-Feigen, hier in Funchal auf Madeira, wirken bisweilen wie Elefantenfüße. (Foto: H. Steinecke)

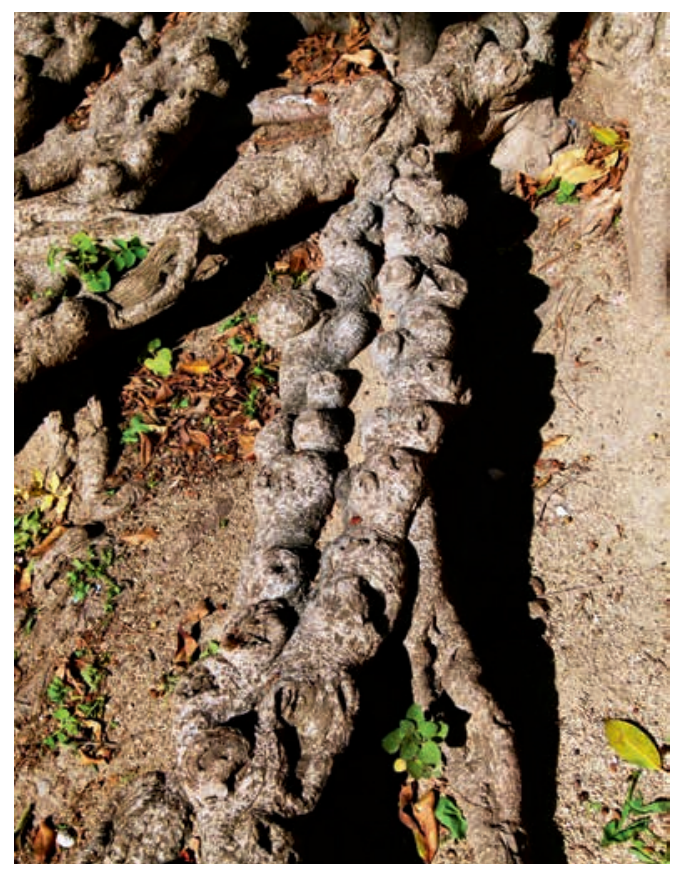

Abb. 10: Manchmal erinnern die über den Boden kriechenden Wurzeln auch an Tintenfischarme.

(Foto: H. Steinecke) 


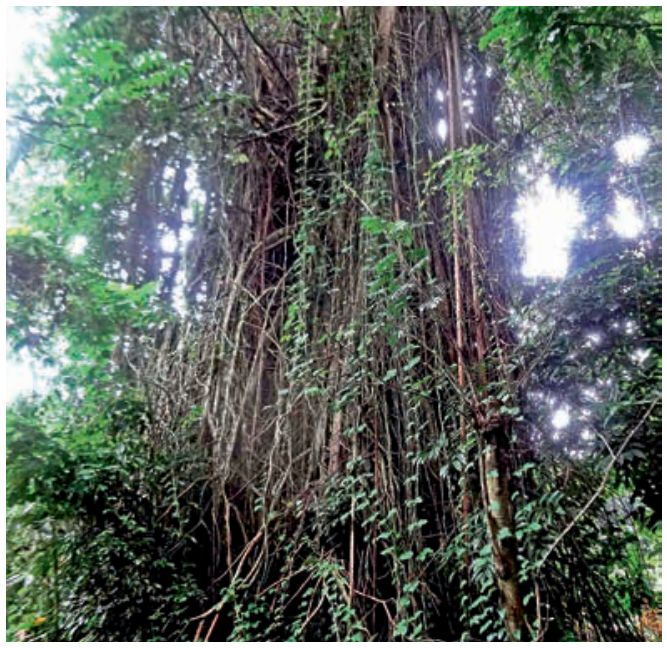

Abb. 11: Nicht nur Ficus-Bäume bilden Luftwurzeln, ebenso der Giftbaum (Antiaris toxicaria), wie Ficus ein Vertreter der Moraceae. (Foto: H. ZAHORKA)

kleine Feigenwespen handelt. Den Zugang in das Innere des Blütenstandes bildet eine kleine Öffnung (Ostiolum) gegenüber dem Ansatz. Um hineinzugelangen, streifen die weiblichen Wespen ihre Flügel und Antennen ab. Im Inneren erfolgt die Bestäubung der Blüten mit den relativ langen Griffeln, aus denen später die winzigen Steinfrüchte entstehen. Nur in die Fruchtknoten der Blüten mit kurzem Griffel kann das Insektenweibchen mit seinem relativ kurzen Legebohrer seine Eier legen. Nach der Eiablage sterben die Tiere. In den Fruchtknoten entwickeln sich die Larven bis zu den fertigen Feigenwespen. Später schlüpfen männliche und weibliche Wespen. Die Männchen begatten noch in der „Feigenfrucht" die Weibchen, fressen ein Loch durch die Feigenwand und sterben danach. Die weiblichen Wespen verlassen, beladen mit befruchteten Eiern und Feigenpollen aus den erst jetzt stäubenden männlichen Blüten, die Feige durch diese freigebissene Öffnung. Sie suchen einen jungen Feigenblütenstand auf, kriechen hinein, bestäuben dabei die Blüten im Innern, legen ihre Eier ab und sterben.

Die Echte Feige (Ficus carica) mit ihren beliebten essbaren Früchten ist keine Würgefeige. Sie wird seit mehr als 2000 Jahren nur vegetativ vermehrt. Die zuckerreichen Fruchtverbände werden frisch oder getrocknet verzehrt. Die dreilappigen Blätter von

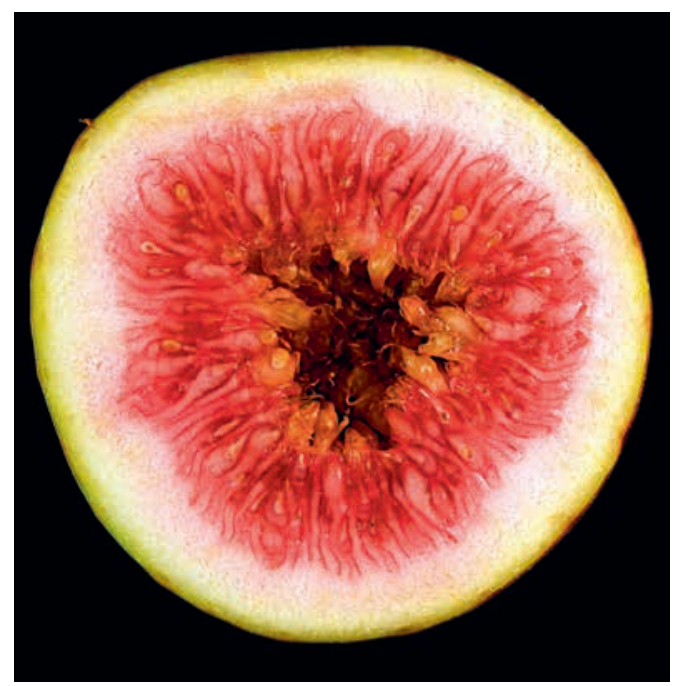

Abb. 12: Querschnitt durch eine Essfeige. (Foto: J. MARKWIRTH)

Ficus carica wurden durch ADAM und Eva bekannt. Echte Feigen sind beliebte Fruchtbäume in wärmeren Ländern, so auch in Italien oder Griechenland. An geschützten Standorten in Deutschland sind sie ebenfalls winterhart. Die Feigen im Palmengarten gedeihen auf dem Mittelmeerhang in der Nähe des Tropicariums und fruchten auch regelmäßig.

\section{Literatur und Internetseiten}

Bolay, E., 1977: Ökologie der Würgefeigen. - Biol. unserer Zeit 2: 55-58.

Zahorka, H. 2000: Das Geheimnis der Pfeilgiftherstellung bei den Blasrohrjägern Borneos - mit Antiaris toxicaria und Licuala spinosa. - Palmengarten 64: 17-22.

Zahorka, H. 2006: Antiaris toxicaria Lesch. 1810. Enzyklopädie der Holzgewächse, 44. Erg.Lfg.6/06, III-4. - Landsberg.

\section{Internetseiten}

https://de.wikipedia.org/wiki/feigen

https://en.wikipedia.org/wiki/Strangler_fig

https://www.britannica.com/plant/strangler-fig-tree www.blueplanetbiomass.org/strangler_figs.htm www.abenteuer-regenwald.de/wissen/pflanzen/wuergefeigen

\section{Anschrift des Autors}

Herwig Zahorka, JI Bondoga Puri Mas C27, Bogor 16131 Indonesien, E-Mail: zahorka.herwig@gmail.com 